\title{
Numerical analysis of non-linear vibrations of a fractionally damped cylindrical shell under the conditions of combinational internal resonance
}

\author{
Yury A. Rossikhin ${ }^{1}$, Marina V. Shitikova ${ }^{1}$, and Basem Ajarmah ${ }^{1,2^{*}}$ \\ ${ }^{1}$ Voronezh State Technical University, Research Center on Dynamics of Solids and Structures, 394006 Voronezh, Russia \\ ${ }^{2}$ Al-istiqlal University Jericho, Palestine
}

\begin{abstract}
Non-linear damped vibrations of a cylindrical shell embedded into a fractional derivative medium are investigated for the case of the combinational internal resonance, resulting in modal interaction, using two different numerical methods with further comparison of the results obtained. The damping properties of the surrounding medium are described by the fractional derivative Kelvin-Voigt model utilizing the Riemann-Liouville fractional derivatives. Within the first method, the generalized displacements of a coupled set of nonlinear ordinary differential equations of the second order are estimated using numerical solution of nonlinear multi-term fractional differential equations by the procedure based on the reduction of the problem to a system of fractional differential equations. According to the second method, the amplitudes and phases of nonlinear vibrations are estimated from the governing nonlinear differential equations describing amplitudeand-phase modulations for the case of the combinational internal resonance. A good agreement in results is declared.
\end{abstract}

\section{Introduction}

Shells are in the art of many structural and building elements due to their several applications. Shell elements are in pipes and ducts, bodies of cars, space shuttles, aircraft fuselages, ship hulls, submarines and building structures. Recent investigations on dynamic response of cylindrical shells are based on nonlinear theories [1-5], and a comprehensive review could be found in $[6,7]$.

Recently Rossikhin and Shitikova [8] proposed a new approach for studying nonlinear dynamic response of thin fractionally damped cylindrical shells embedded into a fractional derivative viscoelastic medium. The dynamic behavior of the shell is described by a set of three coupled nonlinear differential equations The displacement functions are determined in terms of eigenfunctions of linear vibrations. The procedure resulting in decoupling linear parts of equations is proposed with the further use of the method of multiple scales for solving nonlinear governing equations of motion.

This procedure has been utilized for the analytical analysis of free vibrations of cylindrical shell subjected to the conditions of the different internal resonances, resulting in the interaction of two (in the case of two-toone, one-to-one or three-to-one internal resonance $[6,9]$ ) or three modes corresponding to the mutually orthogonal displacements (in the case of combinational internal resonances of additive or difference type [7]).
It has been shown that that the phenomenon of the internal resonance could be very critical, since in a circular cylindrical shell the internal resonance is always present. The influence of viscosity on the energy exchange mechanism has been analyzed. It has been shown that each mode is characterized by its damping coefficient dependent on the natural frequency by the exponential relationship with a negative fractional exponent.

In this paper, we are going to verify parameter values corresponding to the nonlinear vibrations of a fractionally damped cylindrical shell under the conditions of a combinational internal resonance, when a certain natural frequency of vibrations is equal to the sum of two other natural frequencies, using two different numerical methods. In the first method, the generalized displacements of a coupled set of nonlinear ordinary differential equations of the second order are estimated using the numerical solution of nonlinear multi-term fractional differential equations by the procedure based on the reducing of the problem to a system of fractional differential equations $[10,11]$. According to the second method, the amplitudes and phases of nonlinear vibrations are estimated from the governing nonlinear differential equations describing amplitude-and-phase modulations for the case of the combinational internal resonance [7] using the Runga-Kutta fourth order method.

\footnotetext{
* Corresponding author: basem@pass.ps
} 


\section{Problem formulation and governing equations}

Let us consider the dynamic response of a free supported nonlinear elastic circular cylindrical shell of radius $R$ and length $l$, vibrations of which in the cylindrical system of coordinates described by the Donnell-Mushtari-Vlasov equations in terms of three mutually orthogonal displacements [12]:

$\frac{\partial^{2} u}{\partial x^{2}}+\frac{1-v}{2} \frac{1}{R^{2}} \frac{\partial^{2} u}{\partial \varphi^{2}}+\frac{1+v}{2} \frac{1}{R} \frac{\partial^{2} v}{\partial x \partial \varphi}-v \frac{1}{R} \frac{\partial w}{\partial x}+\frac{\partial w}{\partial x} \frac{\partial^{2} w}{\partial x^{2}}$
$+\frac{1+v}{2} \frac{1}{R^{2}} \frac{\partial w}{\partial \varphi} \frac{\partial^{2} w}{\partial x \partial \varphi}+\frac{1-v}{2} \frac{1}{R^{2}} \frac{\partial w}{\partial x} \frac{\partial^{2} w}{\partial \varphi^{2}}=\frac{\rho\left(1-v^{2}\right)}{E} \frac{\partial^{2} u}{\partial t^{2}}$

$\frac{1}{R^{2}} \frac{\partial^{2} v}{\partial \varphi^{2}}+\frac{1-v}{2} \frac{\partial^{2} v}{\partial x^{2}}+\frac{1+v}{2} \frac{1}{R} \frac{\partial^{2} u}{\partial x \partial \varphi}-\frac{1}{R^{2}} \frac{\partial w}{\partial \varphi}+\frac{1}{R^{3}} \frac{\partial w}{\partial \varphi} \frac{\partial^{2} w}{\partial \varphi^{2}}$

$+\frac{1+v}{2} \frac{1}{R} \frac{\partial w}{\partial x} \frac{\partial^{2} w}{\partial x \partial \varphi}+\frac{1-v}{2} \frac{1}{R} \frac{\partial w}{\partial \varphi} \frac{\partial^{2} w}{\partial x^{2}}=\frac{\rho\left(1-v^{2}\right)}{E} \frac{\partial^{2} v}{\partial t^{2}}$

$$
\begin{aligned}
& \frac{h^{2}}{12} \nabla^{4} w+\frac{1}{R^{2}} w-v \frac{1}{R} \frac{\partial u}{\partial x}-\frac{1}{R^{2}} \frac{\partial v}{\partial \varphi}-\frac{1}{2} \frac{v}{R}\left(\frac{\partial w}{\partial x}\right)^{2}-\frac{1}{2} \frac{1}{R^{3}}\left(\frac{\partial w}{\partial \varphi}\right)^{2} \\
& -\frac{\partial}{\partial x}\left[\frac{\partial w}{\partial x}\left(\frac{\partial u}{\partial x}+\frac{v}{R} \frac{\partial v}{\partial \varphi}-\frac{v}{R} w\right)+\frac{1-v}{2} \frac{1}{R} \frac{\partial w}{\partial \varphi}\left(\frac{1}{R} \frac{\partial u}{\partial \varphi}+\frac{\partial v}{\partial x}\right)\right] \\
& -\frac{1}{R} \frac{\partial}{\partial \varphi}\left[\frac{1}{R} \frac{\partial w}{\partial \varphi}\left(v \frac{\partial u}{\partial x}+\frac{1}{R} \frac{\partial v}{\partial \varphi}-\frac{1}{R} w\right)+\frac{1-v}{2} \frac{\partial w}{\partial x}\left(\frac{1}{R} \frac{\partial u}{\partial \varphi}+\frac{\partial v}{\partial x}\right)\right] \\
& =\frac{\rho\left(1-v^{2}\right)}{E} \frac{\partial^{2} w}{\partial t^{2}}
\end{aligned}
$$

where $x$-axis is directed along the axis of the cylinder, $\varphi$ is the polar angle in the plane perpendicular to the $x$-axis, $u=u(x, \varphi, t), v=v(x, \varphi, t)$, and $w=w(x, \varphi, t)$ are the displacements of points located in the shell's middle surface in three mutually orthogonal directions $x, \varphi, r$, $r$ is the polar radius, $h$ is the thickness, $\rho$ is the density, $E$ and $v$ are the elastic modulus and Poisson's ratio, respectively, $t$ is the time, and

$$
\nabla^{4}=\nabla^{2} \nabla^{2}=\frac{\partial^{4}}{\partial x^{4}}+2 \frac{1}{R^{2}} \frac{\partial^{4}}{\partial x^{2} \partial \varphi^{2}}+\frac{1}{R^{4}} \frac{\partial^{4}}{\partial \varphi^{4}} .
$$

The initial conditions

$$
\begin{gathered}
\left.u\right|_{t=0}=\left.v\right|_{t=0}=\left.w\right|_{t=0}, \\
\left.\dot{u}\right|_{t=0}=\varepsilon V_{1}^{0}(x, \varphi),\left.\quad \dot{v}\right|_{t=0}=\varepsilon V_{2}^{0}(x, \varphi),\left.\quad \dot{w}\right|_{t=0}=\varepsilon V_{3}^{0}(x, \varphi
\end{gathered}
$$

where $V_{\alpha}^{0}(x, \varphi)$ are the corresponding initial velocities, and $\varepsilon$ is a small value, should be added to Eqs. (1)-(3). Hereafter over dots denote time-derivatives.

The boundary conditions for a simply supported shell (Navier conditions for the edges free supported in the $x$ direction) have the form [12]:

$$
\begin{gathered}
\left.w\right|_{x=0}=\left.w\right|_{x=l}=0,\left.v\right|_{x=0}=\left.v\right|_{x=l}=0, \\
\left.\frac{\partial^{2} w}{\partial x^{2}}\right|_{x=0}=\left.\frac{\partial^{2} w}{\partial x^{2}}\right|_{x=l}=0,\left.\frac{\partial u}{\partial x}\right|_{x=0}=\left.\frac{\partial u}{\partial x}\right|_{x=l}=0 .
\end{gathered}
$$

Internal resonances, which could occur during free vibrations of elastic circular cylindrical shells described by partial differential equations (1)-(3) with initial and boundary conditions (4)-(6), were revealed and studied in [13] utilizing the solution of the Navier type

$$
\begin{aligned}
& u(x, \varphi, t)=\sum_{m=1}^{\infty} \sum_{n=1}^{\infty} x_{1 m n}(t) \eta_{1 m n}(x, \varphi), \\
& v(x, \varphi, t)=\sum_{m=1}^{\infty} \sum_{n=1}^{\infty} x_{2 m n}(t) \eta_{2 m n}(x, \varphi),
\end{aligned}
$$

(7)

$$
w(x, \varphi, t)=\sum_{m=1}^{\infty} \sum_{n=1}^{\infty} x_{3 m n}(t) \eta_{3 m n}(x, \varphi),
$$

where $\quad x_{\text {imn }}(t)$ and $\eta_{\text {imn }}(x, \varphi)(i=1,2,3) \quad$ are, respectively, the generalized time-dependent displacements and natural functions corresponding to the boundary conditions (6).

During the last decade, fractional calculus entered the mainstream of engineering analysis, and it has been widely applied to structural dynamics problems both in discrete and continuous equations. The results obtained in the field critically estimated in the light of the present view of the place and role of the fractional calculus in engineering problems and practice in the state-of-the-art article by Rossikhin and Shitikova [8].

Rossikhin and Shitikova [8] suggested a new idea to model nonlinear damped vibrations of plates and shells in a viscoelastic medium representing viscous resistance forces via fractional order time derivatives, as distinct to the traditional way [14] when damping forces are assumed to be proportional to first order time-derivatives of displacements. It has been shown in [15] that viscoelastic fractional derivative models used for dynamic response of suspension bridges produce better fit of theoretical investigation with experimental results.

Thus, utilizing the fractional derivative Kelvin-Voigt model and the procedure proposed in [8] for decoupling the linear parts of differential equations of motion of the cylindrical shell (1)-(3), the system of equations of three predominating modes of vibrations, which could be coupled by some conditions of internal resonance, have been derived in $[6,7,9]$ and written in the following dimensionless form:

$$
\begin{aligned}
& \ddot{X}_{1 m n}+\alpha_{1} D^{\gamma} X_{1 m n}+\Omega_{1 m n}^{2} X_{1 m n}=-\sum_{i=1}^{3} F_{i m n} L_{i m n}^{I} \\
& \ddot{X}_{2 m n}+\alpha_{2} D^{\gamma} X_{2 m n}+\Omega_{2 m n}^{2} X_{2 m n}=-\sum_{i=1}^{3} F_{i m n} L_{i m n}^{I I} \\
& \ddot{X}_{3 m n}+\alpha_{3} D^{\gamma} X_{3 m n}+\Omega_{3 m n}^{2} X_{3 m n}=-\sum_{i=1}^{3} F_{i m n} L_{i m n}^{I I I}
\end{aligned}
$$

where $m$ and $n$ are integers defining the number of the natural mode of vibration, $\mathrm{D}^{\gamma}=(d / d t)^{\gamma}$ is the fractional order $(0<\gamma<1)$ of the operator of differentiation $[8,15], \quad \alpha_{i}=\varepsilon \mu_{i} \tau_{i}^{\gamma}(i=1,2,3)$ are coefficients of small viscosity, $\mu_{i}$ are modal damping coefficients, $\tau_{i}$ are modal retardation times, $F_{i m n}$ are nonlinear terms [6].

In equations (8)-(10), $X_{\text {imn }}$ are new generalized displacements which are connected with $x_{i m n}(t)$ via eigenvectors $L_{i m n}^{I}$, $L_{i m n}^{I I}$, $L_{i m n}^{I I I}$ 


$$
x_{i m n}(t)=X_{1 m n} L_{i m n}^{I}+X_{2 m n} L_{i m n}^{I I}+X_{3 m n} L_{i m n}^{I I I}
$$

of the matrix $S_{i j}^{m n}$ with the corresponding eigenvalues $\Omega_{1 \mathrm{mn}}, \Omega_{2 \mathrm{mn}}$, and $\Omega_{3 \mathrm{mn}}$, the elements of which are the following:

$$
\begin{aligned}
& S_{11}^{m n}=\left(\pi^{2} m^{2}+\frac{1-v}{2} \beta_{1}^{2} n^{2}\right), S_{12}^{m n}=S_{21}^{m n}=\frac{1+v}{2} \beta_{1} \pi m n, \\
& S_{13}^{m n}=S_{31}^{m n}=v \beta_{1} \pi m, \quad S_{23}^{m n}=S_{32}^{m n}=\beta_{1}^{2} n, \\
& S_{22}^{m n}=\left(\frac{1-v}{2} \pi^{2} m^{2}+\beta_{1}^{2} n^{2}\right), \\
& S_{33}^{m n}=\frac{\beta_{2}^{2}}{12}\left(\pi^{2} m^{2}+\beta_{1}^{2} n^{2}\right)^{2}+\beta_{1},
\end{aligned}
$$

where $\beta_{1}=l / R$ and $\beta_{2}=h / l$ are the parameters defining the dimensions of the shell.

Method of multiple time scales [16] has been directly applied to the governing differential equations (8)-(10), resulting in six first-order nonlinear ordinarydifferential equations governing the modulation of the amplitudes and phases of the three interacting modes in case of the combinational additive-difference internal resonance $\Omega_{1}=\Omega_{2}+\Omega_{3}$ :

$$
\begin{gathered}
\left(a_{1}^{2}\right)^{\cdot}+s_{1} a_{1}^{2}=\Omega_{1}^{-1} a_{23}^{I} a_{1} a_{2} a_{3} \sin \delta, \\
\left(a_{2}^{2}\right)^{\cdot}+s_{2} a_{2}^{2}=-\Omega_{2}^{-1} a_{13}^{I I} a_{1} a_{2} a_{3} \sin \delta, \\
\left(a_{3}^{2}\right)^{\cdot}+s_{3} a_{3}^{2}=-\Omega_{3}^{-1} a_{12}^{I I I} a_{1} a_{2} a_{3} \sin \delta, \\
\dot{\varphi}_{1}-\frac{1}{2} \sigma_{1}-\frac{1}{2} \frac{a_{23}^{I}}{\Omega_{1}} \frac{a_{2} a_{3}}{a_{1}} \cos \delta=0, \\
\dot{\varphi}_{2}-\frac{1}{2} \sigma_{2}-\frac{1}{2} \frac{a_{13}^{I I}}{\Omega_{2}} \frac{a_{1} a_{3}}{a_{2}} \cos \delta=0, \\
\dot{\varphi}_{3}-\frac{1}{2} \sigma_{3}-\frac{1}{2} \frac{a_{12}^{I I I}}{\Omega_{3}} \frac{a_{1} a_{2}}{a_{3}} \cos \delta=0,
\end{gathered}
$$

where $a_{i}$ and $\varphi_{i} \quad(i=1,2,3)$ are, respectively, amplitudes and phases, $\delta=\varphi_{1}-\left(\varphi_{2}+\varphi_{3}\right)$ is the phase difference, $\psi=\frac{1}{2} \pi \gamma, s_{i}=\mu_{i} \tau_{i}^{\gamma} \Omega_{i}^{\gamma-1} \sin \psi, \sigma_{i}=\mu_{i} \tau_{i}^{\gamma} \Omega_{i}^{\gamma-1} \cos \psi$ , and $a_{23}^{I}, a_{13}^{I I}, a_{12}^{I I I}$ are the coefficients depending on the shell's dimensions, the properties of material it is made of, as well as on the numbers of the interacting modes, which could be found in $[6,7]$.

Thus, the solution of equations (12-17) could provide an approximation solution for small but finite amplitudes weakly varying with time based on a third-order uniform expansion in terms of different time scales. This set of the differential equations in amplitudes and phases approximates the previous system of equations (8-10) in the case of the additive combinational resonance $\Omega_{1}=\Omega_{2}+\Omega_{3}$.

\section{Method of solution}

\subsection{Defining shell's parameters that could satisfy the combinational resonance conditions}

To find the parameters which could satisfy the conditions of the combinational internal resonance $\Omega_{1}=\Omega_{2}+\Omega_{3}$, we should use the property of the symmetric matrix $S_{i j}^{m n}$ possessing three real eigenvalues $\Omega_{i m n} \quad(i=1,2,3)$ corresponding to the three mutually orthogonal eigenvectors:

$$
S_{i j}^{m n}=\Omega_{1 m n}^{2} L_{i m n}^{I} L_{j m n}^{I}+\Omega_{2 m n}^{2} L_{i m n}^{I I} L_{j m n}^{I I}+\Omega_{3 m n}^{2} L_{i m n}^{I I I} L_{j m n}^{I I I} .
$$

The values for $m, n, R, l, h, v$ which satisfy the equality $\Omega_{1}=\Omega_{2}+\Omega_{3}$ for every $m, n$ are presented below in Table 1 .

Reference to Table (1) it shows that the condition of the combinational internal resonance could occur very often, resulting in coupling of three interacting modes of vibrations.

Table 1. Shell parameters that satisfy resonance conditions

\begin{tabular}{|l|l|l|l|l|l|l|l|l|}
\hline $\mathbf{\Omega}_{\mathbf{1}}$ & $\mathbf{\Omega}_{\mathbf{2}}$ & $\mathbf{\Omega}_{\mathbf{3}}$ & $m$ & $n$ & $\mathbf{v}$ & $\boldsymbol{l}$ & $\boldsymbol{h}$ & $\boldsymbol{R}$ \\
\hline 6.55 & 3.83 & 2.72 & 2 & 3 & 0.32 & 0.24 & 0.05 & 0.39 \\
\hline 6.43 & 3.81 & 2.62 & 2 & 3 & 0.3 & 0.23 & 0.05 & 0.5 \\
\hline 6.30 & 3.71 & 2.60 & 2 & 3 & 0.3 & 0.23 & 0.05 & 1.17 \\
\hline 6.28 & 3.64 & 2.64 & 2 & 3 & 0.32 & 0.22 & 0.05 & 2.59 \\
\hline 6.28 & 3.72 & 2.57 & 2 & 3 & 0.3 & 0.24 & 0.05 & 2.89 \\
\hline 6.28 & 3.72 & 2.57 & 2 & 3 & 0.3 & 0.2 & 0.05 & 2.41 \\
\hline
\end{tabular}

\subsection{Numerical solution of general multi-term linear equations}

Using the method proposed in $[10,11,17,18]$, the procedure based on the reduction of the problem to a system of fractional differential equations to estimate numerically the solution of equations $(8-10)$ is as follows:

let

$$
\begin{gathered}
Y_{1}=X_{1}, \\
Y_{2}=D^{\gamma} X_{1}=D^{\gamma} Y_{1}, \\
Y_{3}=D X_{1}=D Y_{1}, \\
\ddot{X}_{1}=D D X_{1}=D Y_{3}
\end{gathered}
$$

and then substitute these equalities in equation (8), resulting in

$$
\begin{gathered}
D Y_{3}=-\sum_{i=1}^{3} F_{1 m n} L_{i m n}^{I}-a_{1} Y_{2}-\Omega_{1}^{2} Y_{1}, \\
Y_{4}=X_{2}, \\
Y_{5}=D^{\gamma} X_{2}=D^{\gamma} Y_{4}, \\
Y_{6}=D X_{2}=D Y_{4}, \\
\ddot{X}_{2}=D D X_{2}=D Y_{6},
\end{gathered}
$$




$$
\begin{gathered}
D Y_{6}=-\sum_{i=1}^{3} F_{2 m n} L_{i m n}^{I I}-a_{2} Y_{5}-\Omega_{2}^{2} Y_{4}, \\
Y_{7}=X_{3}, \\
Y_{8}=D^{\gamma} X_{3}=D^{\gamma} Y_{7}, \\
Y_{9}=D X_{3}=D Y_{7}, \\
\ddot{X}_{3}=D D X_{3}=D Y_{9},
\end{gathered}
$$

and finally in equation (10), resulting in

$$
D Y_{9}=-\sum_{i=1}^{3} F_{3 m n} L_{i m n}^{I I}-\alpha_{3} Y_{8}-\Omega_{3}^{2} Y_{7} .
$$

Thus, the governing set of nine equations in nine unknown values $Y_{i}$ in the matrix form could be written as

$\cdot\left[\begin{array}{ccccccccc}D^{\gamma} & 0 & 0 & 0 & 0 & 0 & 0 & 0 & 0 \\ D & 0 & 0 & 0 & 0 & 0 & 0 & 0 & 0 \\ 0 & 0 & D & 0 & 0 & 0 & 0 & 0 & 0 \\ 0 & 0 & 0 & D^{\gamma} & 0 & 0 & 0 & 0 & 0 \\ 0 & 0 & 0 & D & 0 & 0 & 0 & 0 & 0 \\ 0 & 0 & 0 & 0 & 0 & D & 0 & 0 & 0 \\ 0 & 0 & 0 & 0 & 0 & 0 & D^{\gamma} & 0 & 0 \\ 0 & 0 & 0 & 0 & 0 & 0 & D & 0 & 0 \\ 0 & 0 & 0 & 0 & 0 & 0 & 0 & 0 & D\end{array}\right] *\left[\begin{array}{c}Y_{1} \\ Y_{2} \\ Y_{3} \\ Y_{4} \\ Y_{5} \\ Y_{6} \\ Y_{7} \\ Y_{8} \\ Y_{9}\end{array}\right]$

$$
=\left[\begin{array}{c}
Y_{2} \\
Y_{3} \\
-\sum_{i=1}^{3} F_{1 m n} L_{i m n}^{I I}-c_{1} Y_{2}-\Omega_{1}^{2} Y_{1} \\
Y_{5} \\
Y_{6} \\
-\sum_{i=1}^{3} F_{2 m n} L_{i m n}^{I I}-c_{2} Y_{5}-\Omega_{2}^{2} Y_{4} \\
Y_{8} \\
Y_{9} \\
-\sum_{i=1}^{3} F_{3 m n} L_{i m n}^{I I I}-c_{3} Y_{8}-\Omega_{3}^{2} Y_{7}
\end{array}\right]
$$

Two different types of discretization of derivatives in (19) could be utilized [10, 17]. For the first order differential equation, usually the Trapezium rule is used:

$$
D Y=f \stackrel{\text { yields }}{\rightarrow} Y_{i}=Y_{i-1}+\frac{1}{2} h\left(f_{i}+f_{i-1}\right) .
$$

So in our problem, the discrete derivatives (D) will take the form

$$
Y_{i}-\frac{1}{2} h f_{i}=Y_{i-1}+\frac{1}{2} h\left(f_{i-1}\right) .
$$

To discretize the fractional derivative, the Diethelm's method could be used [10]:

$$
D^{\gamma} Y=\frac{1}{\alpha \gamma_{i}}\left(\sum_{k=0}^{i}{ }^{\alpha} \omega_{k, i} Y_{i-k}+\frac{Y_{0}}{\alpha}\right),
$$

where ${ }^{\alpha} \gamma_{i}=(i h)^{\alpha} \Gamma(-\alpha)$ and ${ }^{\alpha} \omega_{k, 0}, \ldots .,{ }^{\alpha} \omega_{k, i}[10$, 11] are convolution weights derived from the fact that the fractional operator defined in terms of a convolution integral. We will use the weights of the quadrature formula [10]

$$
\begin{aligned}
& f(\alpha)=\alpha(1-\alpha) j^{-\alpha \alpha} \omega_{k, 0}= \\
& =\left\{\begin{array}{lc}
-1 & \text { for } k=0 \\
2 k^{1-\alpha}-(k-1)^{1-\alpha}-(k+1)^{1-\alpha} & \text { for } k=1,2, ., j-1 \\
(\alpha-1) k^{-\alpha}-(k-1)^{1-\alpha}+k^{1-\alpha} & \text { for } k=j
\end{array}\right.
\end{aligned}
$$

Discretization of the equation

$$
D^{\gamma} Y_{1}=Y_{2} \text {. }
$$

results in the following relationships (note $\alpha=\gamma$ ):

$$
\begin{aligned}
& \frac{1}{{ }^{\alpha} \gamma_{i}}\left(\sum_{k=0}^{i}{ }^{\alpha} \omega_{\omega_{k, i}} Y_{1 i-k}+\frac{Y_{10}}{\alpha}\right)=Y_{2}, \\
& \left({ }^{\alpha} \omega_{0, i} Y_{1 i}+\sum_{k=1}^{i}{ }^{\alpha} \omega_{k, i} Y_{1 i-k}+\frac{Y_{10}}{\alpha}\right)={ }^{\alpha} \gamma_{i} Y_{2 i} . \\
& \text { Let } \sum_{k=1}^{i}{ }^{\alpha} \omega_{k, i} Y_{1 i-k}+\frac{Y_{10}}{\alpha}=s_{1 i-1}, \text { so we have } \\
& \left({ }^{\alpha} \omega_{0, i} Y_{1 i}+s_{1 i-1}\right)={ }^{\alpha} \gamma_{i} Y_{2 i}, \\
& s_{1 i}=-{ }^{\alpha} \omega_{0, i} Y_{1 i}+{ }^{\alpha} \gamma_{i} Y_{2 i} .
\end{aligned}
$$

By the trapezium rule we can discretize $\mathrm{Y}_{3}=\mathrm{DY}_{1}$ as

$$
Y_{1 i}=Y_{1 i-1}+\frac{h}{2}\left(Y_{3 i}+Y_{3 i-1}\right) \text {. }
$$

So rearranging terms

$$
Y_{1 i}-\frac{h}{2} Y_{3 i}=Y_{1 i-1}+\frac{h}{2} Y_{3 i-1}=s_{2 i-1}
$$

and utilizing the trapezium rule, we can discretize

$$
\begin{aligned}
& D Y_{3}=-\sum_{j=1}^{3} F_{1} m n L_{j m n}^{I}-a_{1} Y_{2}-\Omega_{1}^{2} Y_{1}, \\
& Y_{3 i}=Y_{3 i-1}+\frac{h}{2}\left[-\sum_{j=1}^{3}\left(F_{1} m n i+F_{1} m n i-1\right) L_{j m n}^{I}\right. \\
& \left.-\propto_{1}\left(Y_{2 i}+Y_{2 i-1}\right)-\Omega_{1}^{2}\left(Y_{1 i}+Y_{1 i-1}\right)\right]
\end{aligned}
$$

Rearranging terms, we have

$$
\left(Y_{3 i}+\frac{h}{2}\left(\propto_{1} Y_{2 i}+\Omega_{1}^{2} Y_{1 i}\right)=Y_{3 i-1}\right.
$$

$$
+\frac{h}{2}\left(-\sum_{j=1}^{3}\left(F_{1 m n i}+F_{1} m n i-1\right) L_{j m n}^{I}-\alpha_{1} Y_{2 i-1}-\Omega_{1}^{2} Y_{1 i-1}\right)
$$

$=s_{3 i-1}$

Repeating these steps (as we have done in Eqs. (24)(33)) for all other values $\left(Y_{4}-Y_{9}\right)$, and arranging them in matrix form, we obtain 


$$
\begin{aligned}
& {\left[\begin{array}{ccccccccc}
-{ }^{\alpha} \omega_{0, i} & { }^{\alpha} \gamma_{i} & 0 & 0 & 0 & 0 & 0 & 0 & 0 \\
1 & 0 & -\frac{h}{2} & 0 & 0 & 0 & 0 & 0 & 0 \\
\frac{\mathrm{h}}{2} \Omega_{1}^{2} & \frac{\mathrm{h}}{2} \mathfrak{x}_{1} & 1 & 0 & 0 & 0 & 0 & 0 & 0 \\
0 & 0 & 0 & -{ }^{\alpha} \omega_{0, i} & \alpha_{\gamma_{i}} & 0 & 0 & 0 & 0 \\
0 & 0 & 0 & 1 & 0 & -\frac{h}{2} & 0 & 0 & 0 \\
0 & 0 & 0 & \frac{\mathrm{h}}{2} \Omega_{2}^{2} & \frac{\mathrm{h}}{2} \mathfrak{x}_{2} & 1 & 0 & 0 & 0 \\
0 & 0 & 0 & 0 & 0 & 0 & -{ }^{\alpha} \omega_{0, i} & { }^{\alpha} \gamma_{i} & 0 \\
0 & 0 & 0 & 0 & 0 & 0 & 1 & 0 & -\frac{h}{2} \\
0 & 0 & 0 & 0 & 0 & 0 & \frac{\mathrm{h}}{2} \Omega_{3}^{2} & \frac{\mathrm{h}}{2} \mathfrak{x}_{3} & 1
\end{array}\right] *} \\
& {\left[\begin{array}{c}
Y_{1 i} \\
Y_{2 i} \\
Y_{3 i} \\
Y_{4 i} \\
Y_{5 i} \\
Y_{6 i} \\
Y_{7 i} \\
Y_{8 i} \\
Y_{9 i}
\end{array}\right]=\left[\begin{array}{l}
s_{1 i-1} \\
s_{2 i-1} \\
s_{3 i-1} \\
s_{4 i-1} \\
s_{5 i-1} \\
s_{6 i-1} \\
s_{7 i-1} \\
s_{8 i-1} \\
s_{9 i-1}
\end{array}\right]}
\end{aligned}
$$

It is quite straightforward to solve (34).

3.3. Numerical solution of the combinational additive-difference internal resonance using the Runga-Kutta fourth order method

To utilize the Runga-Kutta fourth order method to estimate numerically the solution of equations (12)-(17), we first rewrite these equations in the first order form as follows:

$$
\begin{gathered}
\dot{a}_{1}=\frac{1}{2}\left(\Omega_{1}^{-1} a_{23}^{I} a_{2} a_{3} \sin \delta-s_{1} a_{1}\right), \\
\dot{a}_{2}=\frac{1}{2}\left(\Omega_{2}^{-1} a_{13}^{I I} a_{1} a_{3} \sin \delta-s_{2} a_{2}\right), \\
\dot{a}_{3}=\frac{1}{2}\left(\Omega_{3}^{-1} a_{12}^{I I I} a_{1} a_{2} \sin \delta-s_{3} a_{3}\right), \\
\dot{\varphi}_{1}=\frac{1}{2} \sigma_{1}+\frac{1}{2} \frac{a_{23}^{I}}{\Omega_{1}} \frac{a_{2} a_{3}}{a_{1}} \cos \delta, \\
\dot{\varphi}_{2}=\frac{1}{2} \sigma_{2}+\frac{1}{2} \frac{a_{13}^{I I}}{\Omega_{2}} \frac{a_{1} a_{3}}{a_{2}} \cos \delta, \\
\dot{\varphi}_{3}=\frac{1}{2} \sigma_{3}+\frac{1}{2} \frac{a_{12}^{I I I}}{\Omega_{3}} \frac{a_{1} a_{2}}{a_{3}} \cos \delta .
\end{gathered}
$$

\section{Numerical results}

The numerical solution of equation (34) has been carried out at the dimensionless parameters presented in Table 2 .

Table 2. Shell's parameters.

\begin{tabular}{|l|l|l|l|l|l|l|}
\hline$v$ & $R$ & $l$ & $h$ & $\gamma$ & $m$ & $N$ \\
\hline 0.3 & 2 & 0.28 & 0.042 & $0 \longrightarrow 1$ & 3 & 1 \\
\hline
\end{tabular}
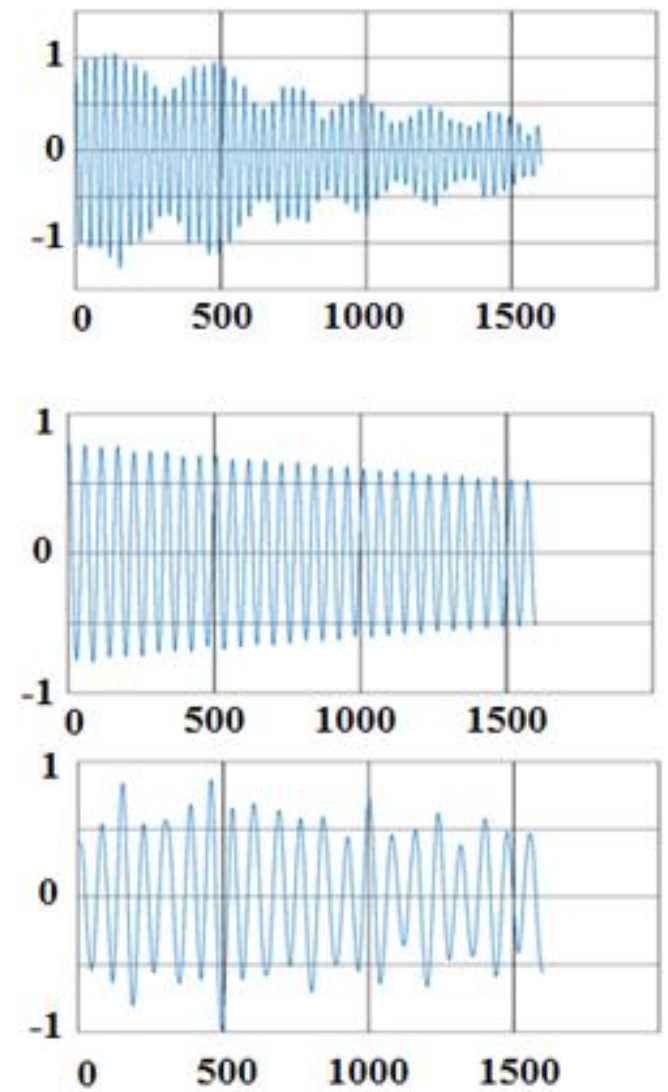

Fig. 1 Plot of numerical solution of equations (34) $X_{1}, X_{2}$, $X_{3}$

Variation of the fractional parameter from $\gamma \rightarrow 0$ to $\gamma \rightarrow 1$ allows one to investigate vibrations of cylindrical shells in surrounding media with different viscous properties, including the pure elastic case at $\gamma=0$ and conventional Kelvin-Voigt model at $\gamma \rightarrow 1$. 


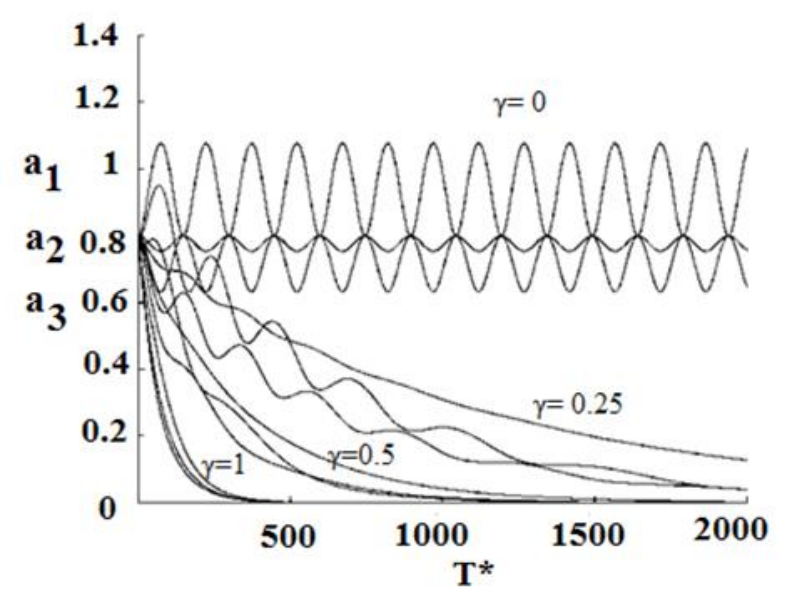

Fig. 2 Dimensionless amplitude vs. dimensionless time as the solution of equations (35-40).

The dynamic behavior of a cylindrical shell in a viscous medium at $\gamma=0.25,0.5$ and 1 , which is found by using the first and second methods, is shown in Fig. 1 and 2, respectively, for the parameters taken from Table 2 , which correspond to the combinational internal resonance at $\Omega_{1}=\Omega_{2}+\Omega_{3}=9.4259=3.8494+5.5764$.

The behavior of amplitudes of vibrations reveals the exchange of energy between the generalized displacements of the system under the considered case of the combinational internal resonance.

\section{Conclusion}

Free damped vibrations of a shallow nonlinear thin cylindrical shell in a fractional derivative viscoelastic medium are investigated numerically by two different methods based on the new approach proposed in [6].

The numerical solutions of the damped vibrations of the nonlinear cylindrical shell subjected to the conditions of the internal resonance have been estimated, and good agreement between the two methods has been achieved. Within the first method, the generalized displacements of a coupled set of nonlinear ordinary differential equations of the second order are calculated using the numerical solution of nonlinear multi-term fractional differential equations by the procedure based on the reducing the problem to a system of fractional differential equations.

According to the second method, the amplitudes and phases of nonlinear vibrations are estimated from the governing nonlinear differential equations describing amplitude-and-phase modulations for the case of the combinational internal resonance.

It has been shown that, as in [6,7], the phenomenon of the internal resonance could be very critical, since in a circular cylindrical shell the internal additive and difference combinational resonances are always present. The effect of viscosity on the energy exchange mechanism is analyzed.
This research was made possible by Grant No. 9.5138.2017/8.9 as a Government task from the Ministry of Education and Science of the Russian Federation.

\section{References}

1. V.D. Kubenko, P.S. Koval'chuk, Int. Appl. Mech. 34, 703-728 (1998)

2. M. Amabili, M.P. Paidoussis, Appl. Mech. Rev. 56, 349-381 (2003)

3. Y.-S. Lee, Trans. KSME 33, 1-26 (2009)

4. I.D. Breslavsky, K.V. Avramov, Meccanica 46, 817-832 (2011)

5. K.V. Avramov, Yu.V. Mikhin, E. Kurilov, Nonlinear Dyn. 47, 331-352 (2012)

6. Yu.A. Rossikhin, M.V. Shitikova, Chapter 17 in: Shell and Membrane Theories in Mechanics and Biology: From Macro- to Nanoscale Structures (H. Altenbach, G.I. Mikhasev, eds., Springer, pp. 259280, 2015) DOI 10.1007/978-3-319-02535-3_17

7. Yu.A. Rossikhin, M.V. Shitikova, Chapter 3 in: Computational Problems in Science and Engineering (N. Mastorakis et al. eds.), Lecture Notes in Electrical Engineering 343, 59-107 (Springer Int. Publ., Switzerland, 2015) DOI 10.1007/978-3-319-15765-8_3

8. Yu.A. Rossikhin, M.V. Shitikova, Appl. Mech. Rev. 63, 010801 (2010)

9. Yu.A. Rossikhin, M.V. Shitikova, Appl. Math. Comp. 257, 498-525 (2015)

10. K. Diethelm, Elect. Trans. Num. Anal. 5, 1-6 (1997)

11. J.T. Edwards, N.J. Ford, A.C. Simpson, J. Comp. Appl. Math. 148, 401-418 (2002)

12. A. Volmir, Nonlinear dynamics of plates and shells (Nauka, Moscow, 1972)

13. Yu.A. Rossikhin, M.V. Shitikova, In: Proceedings of the $18^{\text {th }}$ International Congress on Sound and Vibration 4, 2775-2782, July 11-14, 2011, Rio de Janeiro, Brazil (2011)

14. R.W. Clough, J. Penzien, Dynamics of structures (McGraw-Hill, New York, 1975)

15. Yu.A. Rossikhin, M.V. Shitikova, ASCE J. Eng. Mech, 124, 1029-1036 (1998)

16. A. Nayfeh, Perturbation methods (Wiley, New York 1973)

17. D. Baleanu, K. Diethelm, E. Scalas, J.J. Trujillo, Fractional calculus: Models and numerical methods (World Scientific, Singapore, pp. 88-114, 2012)

18. K. Diethelm, Hildesheimer Informatikberichte (1995) 